\title{
A full study is in progress, but who pays after the pilot?
}

\author{
$\mathrm{n}$ the beginning, everything about \\ Dr. Tonya Abraham's protocol was \\ unremarkable. She was requesting 130 \\ rats for a study to explore the feasibility of \\ using an antibody to interrupt a metabolic \\ pathway associated with chemically induced \\ colon cancer. The study was internally \\ funded by Great Eastern University's \\ Department of Oncology and the \\ department chairman had signed off on the \\ protocol, indicating that he had reviewed \\ and approved it. The protocol stated that \\ a full study with 120 rats would only be \\ performed if an initial pilot study with \\ 10 rats showed promise. The study with \\ 130 rats was approved by the Institutional \\ Animal Care and Use Committee (IACUC) \\ and after the pilot study was completed, \\ and following the standard procedure of the \\ IACUC, the committee reviewed the results \\ of the pilot study and agreed with Abraham \\ that the full study with 120 rats could \\ proceed as per the protocol.
}

A few weeks later, with the full study already in progress, the department chairman was routinely reviewing his department's monthly research expenses when he saw the charge for the 120 rats and associated per diem charges. He asked Abraham for an explanation, which she provided, but much to her surprise the chairman criticized her for spending money he had not approved. It soon became clear that there was a serious misunderstanding. The chairman thought he was approving the pilot study and the full study would be performed only if Abraham could attract a research grant to support it. Abraham thought the chairman was approving both the pilot and the full study, and she would submit a grant for funding future research based on the results of the current study. The immediate problem was that there were insufficient departmental funds to support the expensive ongoing study. This information was transmitted to the IACUC office and the vivarium. The department chairman wanted the study to stop before additional expenses were incurred. Abraham asked the Institutional Official to help fund the study, but he washed his hands of the matter, saying that it was the department's problem. The attending veterinarian felt that stopping the study would waste the lives of all 120 animals, and Tonya Abraham, who was a new assistant professor and principal investigator, was too afraid and upset to even voice an opinion. How would you suggest resolving this problem?

\section{Jerald Silverman}

University of Massachusetts Medical School, Worcester, Massachusetts, USA.

e-mail: Jerald.Silverman@umassmed.edu

Published online: 18 April 2019

https://doi.org/10.1038/s41684-019-0284-Z

\section{Money, mentorship and leadership}

U niversity research programs are complex. Occasional miscommunication is inevitable. In this case, there are multiple interested parties who need to work together to resolve the current situation.

The Institutional Animal Care and Use Committee (IACUC) reviewed and approved the animal use protocol after due diligence, which included a pilot study component. Funding questions are left to the institution to manage, and even the PHS Policy requirement for congruency confirmation $^{1}$ is not prescribed as an IACUC function. Though many institutions do place some oversight of the funding component under the IACUC's authority, this is not a requirement. While the IACUC should be aware of problematic situations, this particular discussion is not within the scope of the IACUC's responsibilities. Understanding that the IACUC has no official authority over fiscal matters, the IACUC nevertheless has an interest in minimizing negative impact to the animals used in the research study and wastage of animals in general. The Institutional Official (IO) does have the authority to allocate resources ${ }^{2}$. Those resources are typically focused on use at a programmatic level as opposed to an individual project.

With detailed knowledge of past and existing animal work at the institution, the IACUC may be able to help Abraham identify an on-campus collaborator who has a similar research focus to take over all or part of the fiscal responsibility for the ongoing study. Other internal funding mechanisms may exist with provisions for emergency funding to buy some time for a more permanent solution. Great Eastern University's research development office should be consulted as they will have information available on a wide variety of funding sources and may be able to identify additional resources.

In the scenario presented, the animals would have to be euthanized if alternate funding for animal husbandry as well as that required for conducting terminal studies cannot be found within a short period of time. Abraham could consider alternate early timelines that would still provide some pertinent information to help inform future funding proposals. Since truncated timelines would result in less pain or distress to the animals, modifications to the protocol could qualify for approval through the veterinary verification and consultation (VVC) ${ }^{3}$ process, if Great Eastern University recognizes this method of review.

Once the immediate issue regarding the 120 rats currently on study is addressed, measures to prevent a recurrence need to be considered. It is evident that there was poor communication between the Department Chair and the new faculty member with respect to expectations and financial support afforded to Abraham. Regardless of the root cause, an improved stream of communication needs to be established. Mentorship is a recognized method of facilitating 